\title{
Capítulo 16
}

\section{Simulação computacional do}

sistema de emissão de ordens CONWIP aplicada em uma empresa automobilística

Amanda Cristie Nobre ${ }^{1}$

Stella Jacyszyn Bachega²

Resumo: Os sistemas de emissão de Ordens (SEO's), ou ordering systems, são responsáveis pelo controle das ordens de produção e compras no chão de fábrica. Dentre os SEO's está o CONWIP. Uma técnica que auxilia as empresas a analisar o desempenho em diversas áreas, como a manufatura, é a simulação computacional. O objetivo deste artigo é simular e analisar o desempenho do CONWIP aplicado em uma empresa do setor automobilístico. Para tanto, utilizou-se a abordagem de pesquisa quantitativa e o procedimento de pesquisa experimental. As medidas de desempenho analisadas foram o total de eixos produzidos, o tempo de processamento de um eixo, as utilizações de cada funcionário e de cada estação de trabalho. Dentre os resultados, observou-se que os estoques intermediários obtiveram baixa utilização, o que indica que os eixos praticamente não esperam nos estoques para serem processados. Esta pode ser considerada uma vantagem do sistema CONWIP.

Palavras-chave: Sistemas de Emissão de Ordens. CONWIP. Simulação Computacional.

1 Universidade Federal de Goiás - UFG. Regional Catalão, Unidade Acadêmica Especial de Engenharia. Contato: amandacristienobre@hotmail.com. Bolsista do Programa Institucional de Bolsa de Iniciação Científica (PIBIC/UFG/CNPq).

2 Universidade Federal de Goiás - UFG. Regional Catalão, Unidade Acadêmica Especial de Engenharia. Contato: stella@ufg.br. 


\section{Introdução}

O Planejamento e Controle da Produção (PCP), quando aliado à técnica de simulação computacional, pode gerar vantagens competitivas para as empresas, devido a melhoria do desempenho destas. A simulação propicia maior conhecimento das atividades desenvolvidas e representadas no modelo. No entanto, segundo Harrel, Ghosh e Bowden (2012), há muita resistência por parte das empresas em utilizar a simulação, devido ao pensamento errado de que esta custaria muito e haveria um consumo de tempo muito grande, sendo que na realidade o que ocorre é o contrário. Mediante o uso da simulação, geralmente ocorre ganho de tempo e menos custos são consumidos.

As áreas de aplicação da simulação são diversas e podem ser divididas em aplicações na manufatura e em serviços. A área de manufatura é o maior campo de aplicação da simulação depois da área militar. Alguns usos práticos na manufatura são: sistemas de movimentação e armazenagem de materiais, linhas de montagem, células automatizadas, problemas de programação da produção e análises de estoque (CHWIF; MEDINA, 2015) e, também, para análise de sistemas de emissão de ordens (LEE; SEO, 2016).

Os sistemas de emissão de ordens (SEO) são denominados por Burbidge (1990) como ordering systems e são classificadas por este como: sistemas para fazer de acordo com o pedido; sistemas de estoques controlados e sistemas de fluxo controlado. O sistema foco deste trabalho, o CONWIP (Constant Work in Process), é classificado como sistemas de estoques controlados. Neste tipo de sistema, há a combinação de produção empurrada entre as estações de trabalho e puxada de modo geral.

Com base neste contexto, o objetivo do presente artigo é simular e analisar o desempenho do CONWIP aplicado em uma empresa do setor automobilístico. Tal pesquisa justifica-se pela importância do tema e pela necessidade de análise do comportamento do sistema real antes de sua efetiva alteração. Ainda, na literatura da área há várias discussões teóricas ou comparações hipotéticas entre SEO’s. Este artigo traz uma aplicação do sistema CONWIP em um ambiente real.

Destaca-se que esta pesquisa possui vínculo com o Grupo de Estudos em Modelagem e Simulação (GEMS) e que faz parte do projeto de pesquisa intitulado "Método para escolha de sistemas de coordenação de ordens com uso de simulação: aplicação em ambientes de produção flow shop".

Para alcançar o objetivo proposto, o artigo está estruturado da seguinte forma: a seção um é destinada para ressaltar as características teóricas do CONWIP; a seção dois trata da metodologia; a seção três apresenta a discussão e os resultados alcançados na pesquisa e, por fim, há as considerações finais. 


\section{Referencial teórico}

Segundo Spearman, Woodruff e Hopp (1990), o CONWIP (Constant Work in Process) é apropriado para ambientes com sistemas puxados e apresenta melhor desempenho em ambientes flow shop e job shop. O sistema de emissão de ordens CONWIP trabalha com ordens controladas por cartões que, segundo Spearman et al. (1990), não estão associados com a quantidade de produtos, mas à linha de produção como um todo.

O CONWIP é considerado por Hopp e Spearman (2008) como um sistema híbrido, pois combina aspectos das estratégias puxadas e empurradas, de forma que a alta taxa de transferência é devido às características empurradas e o controle de WIP (Work in Process) se dá pelo comportamento de sistema puxado. O controle de WIP se dá apenas dentro da linha de produção, enquanto que entre os postos de trabalho o mesmo não ocorre. Seus cartões de produção estão associados à linha de produção e não a determinado número de peças, pois a quantidade de peças é determinada ao início da linha, de acordo com uma lista de pedidos (backlog list). Quanto ao funcionamento do CONWIP, este está representado na Figura 16.1.

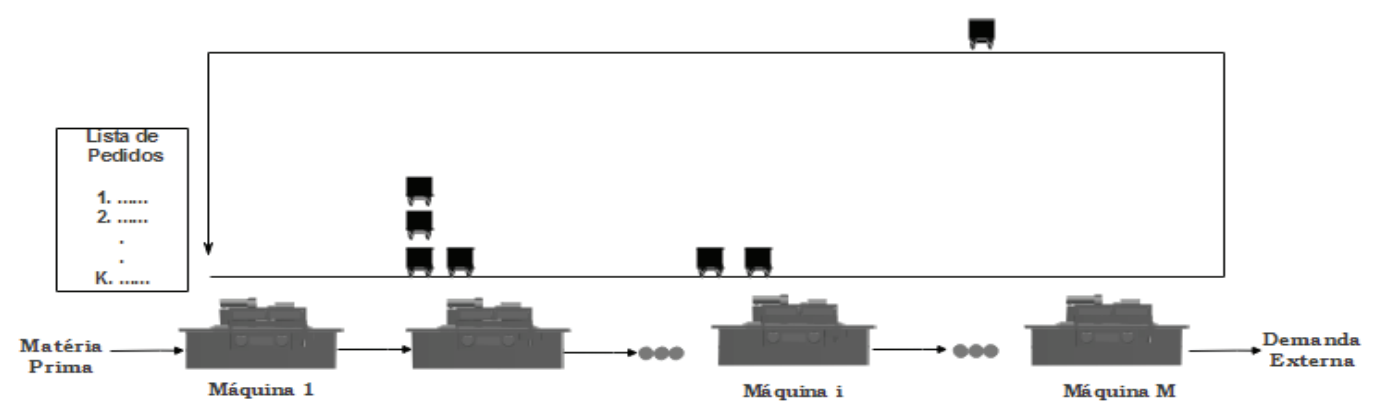

Figura 16.1 Funcionamento do CONWIP

Fonte: Adaptado de Herer e Masin (1997).

Observa-se na Figura 16.1 que: i) um cartão é unido a um contenedor padrão de peças no começo da linha, seguindo a ordem que consta na lista de pedidos em carteira (backlog list); ii) o contenedor movimenta-se ao longo da linha de produção e as peças são processadas de acordo com as necessidades em cada estação de trabalho da linha de produção (Na Figura 16.1 são representadas por Máquina 1, Máquina i e Máquina M); iii) ao final da linha, quando o contenedor é utilizado, o cartão é retirado e volta para o início da linha, onde aguarda em uma fila de cartões para seja anexado a outro contenedor de peças. 
O CONWIP pode ser entendido como um caso especial do sistema kanban, segundo Gong, Yang e Wang (2014). No entanto, ao comparar os dois sistemas, Spearman e Zazanis (1992) constataram que a utilização do sistema CONWIP é mais simples, devido ao fato deste determinar o WIP de forma global, para a linha inteira. Já o kanban especifica uma quantidade de cartões para cada estação.

Conforme Gong, Yang e Wang (2014), a informação de demanda é transferida da última estação de trabalho para a primeira. Ovalle e Marquez (2003) explicam que ao iniciar a produção, todos os cartões estão no começo da linha e à medida que chegam ordens de produção esses vão sendo anexados a elas e prosseguem por todo o tempo de processamento juntos. Ao final do processo, os cartões são encaminhados para o início da linha. Além disso, nenhuma ordem de produção é autorizada para entrar na linha sem o acompanhamento de um respectivo cartão. Assim, quando não se tem cartões disponíveis, as ordens se acumulam até que algum cartão seja disponibilizado. Os estoques intermediários, assim como as ordens de produção, seguem a disciplina das filas FIFO (PEPS).

O CONWIP, de acordo com Sipper e Bulfin Jr. (1997), é uma abordagem que se aplica melhor em linhas de produção em que o fluxo possui estabilidade e uniformidade, além disso, pode ser utilizado em ambientes produtivos com maior variedade de produtos em relação ao kanban.

Algumas vantagens do CONWIP podem ser explicitadas, quando comparado aos sistemas puxados, a saber (OVALLE; MARQUEZ, 2003, p.197): i) por possuir apenas uma configuração para a contagem de cartão, se apresenta de forma mais simples que sistemas com a contagem de cartões a cada posto de trabalho; ii) em relação a prazos e tempos de ciclo, o CONWIP se mostra superior aos demais sistemas de emissão de ordens; iii) o nível de estoque médio do CONWIP supera o nível do kanban, quando comparados no mesmo nível de produção e serviço; iv) o CONWIP tem produzido um throughput médio maior (conforme Hopp e Spearman (2008), throughput refere-se as saídas médias de um processo produtivo por unidade de tempo), menor variação e um volume menor de estoque máximo quando comparado ao kanban em uma linha com produção de apenas um item, entre outras.

Já em relação aos sistemas empurrados, Ovalle e Marquez (2003, p.198) apresentam vantagens do CONWIP, dentre elas: i) quando o sistema de produção opera com níveis de throughput maiores possíveis, o CONWIP se mostra superior aos sistemas empurrados; ii) em comparação com o MRP, o CONWIP obtém menores lead times, os níveis de serviços são melhores e o WIP de produto acabado, além dos estoques, são menores.

Ovalle e Marquez (2003, p. 198) também exibem as seguintes desvantagens do CONWIP: i) não avalia o impacto que um gargalo pode ter no desempenho de uma linha de produção; ii) nem sempre produz o menor número total de movi- 
mentações entre os estágios de produção; iii) pode exigir um local maior para armazenagem entre os estágios do que o kanban, uma vez que os contenedores cheios podem aglomerar-se entre qualquer par de fases alternadas.

Diversos autores realizam estudos sobre o CONWIP, como Jaegler, Burlat e Lamouri (2016), que trazem uma revisão de literatura sobre esse sistema. Huang et al. (2016a) propõem o CONWIP design framework (CDF), que é uma abordagem sistemática de projeto para obter políticas CONWIP alternativas, considerando a perspectiva de estrutura em ciclo. Huang et al. (2016b) apresentam uma abordagem para projetar o ciclo CONWIP para sistema de montagem em ambiente de produção one-of-a-kind (executado sob encomenda específica). Han e Wang (2016) projetaram um sistema de cartões CONWIP eletrônicos, utilizando RFID (Radio Frequency Identification).

\section{Metodologia}

A abordagem de pesquisa utilizada foi a quantitativa, de acordo com Bryman (1989). Nesta pesquisa a abordagem quantitativa foi usada devido a característica da forma de manipulação dos dados e da análise dos resultados gerados.

Quanto ao procedimento de pesquisa, foi empregado a pesquisa experimental, conforme as instruções de Bryman (1989). Geralmente, a pesquisa experimental está associada a experimentos controlados no laboratório ou em modelagens matemáticas e simulações computacionais, a qual esta foi aqui utilizada.

A simulação realizada neste trabalho foi do tipo matemática e executada segundo Law e Kelton (2000), de forma numérica e estocástica. A simulação computacional foi realizada com o auxílio do software ProModel ${ }^{\circledR}$ Professional SP4 versão $8.6^{3}$. Para condução de um estudo em simulação tomou-se como base os passos propostos por Law e Kelton (2000), a saber:

- Etapa 1: Formulação do problema e planejamento do estudo;

- Etapa 2: Coleta de dados e definição dos modelos;

- Etapa 3: Validação dos modelos conceituais;

- Etapa 4: Construção do programa computacional e verificação;

- Etapa 5: Realização de execuções piloto;

- Etapa 6: Validação dos modelos programados;

- Etapa 7: Projeto dos experimentos;

- Etapa 8: Realização das execuções de simulação;

- Etapa 9: Análise de resultados;

- Etapa 10: Documentação e implementação.

3 Fonte: <http://www.belge.com.br/promodel.php>. Acesso em: 27 de out. de 2016. 
Os dados para elaboração do modelo foram coletados em uma empresa do setor automobilístico. Os dados coletados foram a quantidade de estações de trabalho, o tamanho da ordem de produção, a quantidade de funcionários na parte da linha estudada, os tempos de operação em cada estação de trabalho, a jornada de trabalho.

Os tempos de operação foram representados por meio da distribuição Triangular. A escolha desta distribuição deve-se ao fato de não se conhecer a forma exata da distribuição. No entanto, as estimativas para o menor valor, o valor mais provável de ocorrer e o maior valor eram conhecidos (CHWIF; MEDINA, 2015). Destaca-se que somente para a estação de trabalho 6 o tempo de operação é constante, independentemente do tipo de eixo produzido, devido a características da linha de produção. Para a representação do processo de chegada, foram considerados a ordem de ocorrência, os tempos estabelecidos pela empresa e as quantidades de cada eixo presentes na ordem de produção disponibilizada.

Freitas Filho (2008) classifica os sistemas reais como sistemas terminais ou não terminais. Os sistemas terminais, segundo o autor, são aqueles que possuem condições iniciais fixas determinadas e um evento que estabelece o fim natural para a simulação. Este foi o tipo de sistema considerado para se fazer a modelagem, simulação e as análises. Assim, o intervalo de confiança utilizado foi no nível de $95 \%$. Para fazer a interpretação do semi-intervalo de confiança (half-width) tomou-se como base Kelton, Sadowski e Sadowski (2002) que afirmam que em $95 \%$ das replicações os valores obtidos estarão em um intervalo da média \pm o semi-intervalo.

\section{Discussão e resultados}

Nesta seção, apresentam-se as características do modelo elaborado. Ainda, os desempenhos obtidos por meio da aplicação do CONWIP na parte da linha de produção pesquisada são expostos e discutidos.

\subsection{Características do modelo elaborado}

O CONWIP foi simulado nesta pesquisa a partir de dados coletados em uma empresa do setor automobilístico e apenas parte de uma linha de produção foi estudada, que é referente à montagem de eixos traseiros. Vale ressaltar que o CONWIP não está implantado na parte da linha em questão. As características do sistema simulado podem ser observadas na Figura 16.2, a qual representa o cenário CONWIP elaborado no simulador ProModel ${ }^{\circledR}$. 


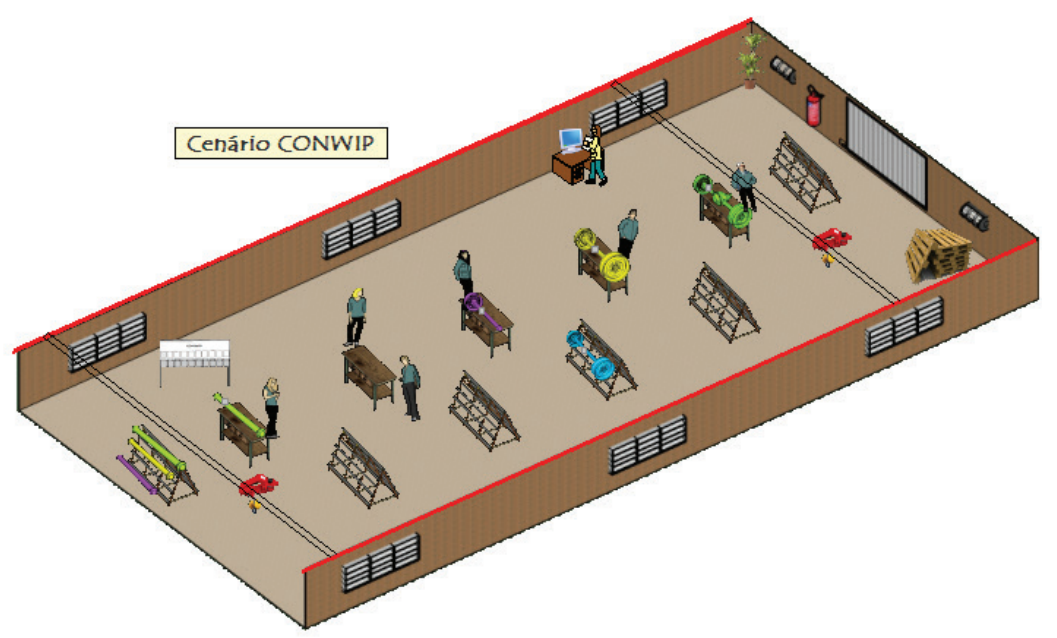

Figura 16.2 Representação do cenário CONWIP

Fonte: Dados da pesquisa.

Nessa linha estão dispostas cinco estações de trabalho e quatro estoques intermediários, utilizados apenas quando a estação seguinte não tem capacidade para processar o eixo. Ainda, há um estoque inicial e um estoque final. Cada estação de trabalho é capaz de montar um eixo por vez. Para isso, estão presentes seis operadores, sendo um para cada estação de trabalho e apenas dois são alocados na estação de trabalho 2 .

Também, foi representado um posto de cartões CONWIP no início da linha. Há duas talhas elétricas que auxiliam na movimentação das entidades em partes do ambiente produtivo. Cada estação de trabalho é responsável por uma atividade de montagem, até que o eixo esteja pronto no estoque final. As mudanças também podem ser vistas na Figura 16.2. Ainda, podem ser observadas as cores diferentes de eixos na linha de produção, que representam os tipos diferentes de eixos.

A ordem de produção utilizada no modelo é constituída de 130 eixos traseiros, com sete modelos diferentes e quantidades diferentes de cada tipo de eixo. Com base nas informações coletadas, o dimensionamento do posto de cartões foi feito por meio da seguinte equação, conforme Spearman, Woodruff e Hoop (1990) apud Huang, Wang e Ip (1998):

$$
\theta=\mu t
$$

Onde:

- $\theta$ : Representa a quantidade média de cartões; 
- $\mu$ : Representa o throughput médio da linha de produção, ou seja, a taxa de média de saída;

- $t$ : É referente ao tempo médio que um cartão leva para passar por toda linha de produção.

O número de cartões obtido foi de 5,961 cartões. Sipper e Bulfin Jr. (1997) informam que o número de cartões deve o inteiro superior. Assim, o número de cartões CONWIP utilizado no modelo foi de 6 cartões.

\subsection{Desempenho obtido no cenário CONWIP}

Por meio da simulação realizada, é possível analisar o desempenho do sistema CONWIP aplicado em um ambiente real. Para esta análise, foram utilizados os valores médio, mínimo, máximo, desvio padrão e semi-intervalo (half-width). Este último foi utilizado para poder afirmar se a quantidade de replicações executadas foi satisfatória para a análise dos valores médios obtidos.

Conforme Freitas Filho (2008), se o valor do half-width estiver igual ou abaixo de $10 \%$ da média amostral, o total de replicações é considerado satisfatório. Neste estudo, foram utilizadas 75 replicações e todas as variáveis obtiveram half-width inferior a $10 \%$ da média, portanto, a quantidade utilizada de replicações foi satisfatória.

A Tabela 16.1 aborda a quantidade produzida de cada tipo de eixo traseiro. Em 8,63 horas de simulação, que representam as horas úteis na linha analisada para a jornada de um dia de trabalho, o sistema CONWIP foi capaz de produzir em média 94,52 eixos.

Ainda conforme a Tabela 16.1, o eixo mais produzido durante a simulação foi o eixo do tipo 5, sendo produzido em média 36,09 eixos, enquanto que o de menor produção média foi o eixo do tipo 1, com o total de 3 eixos. Através do desvio padrão é possível notar que não houve variação entre os valores médios, mínimos e máximos para os eixos 1, 2, 3 e 7 .

Tabela 16.1 Quantidade de eixos produzidos (unidades) no cenário CONWIP

\begin{tabular}{|l|c|c|c|c|c|}
\hline $\begin{array}{c}\text { Tipos de } \\
\text { eixos }\end{array}$ & Média & Mínimo & Máximo & $\begin{array}{c}\text { Desvio } \\
\text { Padrão }\end{array}$ & $\begin{array}{c}\text { Half- } \\
\text { width }\end{array}$ \\
\hline Eixo 1 & 3,00 & 3,00 & 3,00 & 0,00 & 0,00 \\
\hline Eixo 2 & 6,00 & 6,00 & 6,00 & 0,00 & 0,00 \\
\hline Eixo 3 & 4,00 & 4,00 & 4,00 & 0,00 & 0,00 \\
\hline Eixo 4 & 26,43 & 26,00 & 27,00 & 0,50 & 0,11 \\
\hline
\end{tabular}


Tabela 16.1 Quantidade de eixos produzidos (unidades) no cenário CONWIP (Continuação)

\begin{tabular}{|c|c|c|c|c|c|}
\hline $\begin{array}{c}\text { Tipos de } \\
\text { eixos }\end{array}$ & Média & Mínimo & Máximo & $\begin{array}{c}\text { Desvio } \\
\text { Padrão }\end{array}$ & $\begin{array}{c}\text { Half- } \\
\text { width }\end{array}$ \\
\hline Eix0 5 & 36,09 & 35,00 & 38,00 & 0,72 & 0,17 \\
\hline Eix06 & 11,00 & 10,00 & 12,00 & 0,28 & 0,07 \\
\hline Eixo 7 & 8,00 & 8,00 & 8,00 & 0,00 & 0,00 \\
\hline $\begin{array}{c}\text { Total } \\
\text { produzido }\end{array}$ & 94,52 & 92,00 & 98,00 & 1,50 & 0,35 \\
\hline
\end{tabular}

Fonte: Dados da pesquisa.

Nota-se que o valor médio de produção obtido está abaixo do desejado, uma vez que a ordem de produção possuía 130 eixos. Assim, se o foco da empresa for aumentar o total diário de saídas do sistema, recomenda-se testar cenários com outros sistemas de emissão de ordens, antes da tomada de decisão final sobre a implantação do CONWIP.

A Tabela 16.2 apresenta o tempo médio, em minutos, que um eixo fica no sistema, a contar desde a sua entrada na parte da linha de produção até a sua saída. Dessa forma, um eixo fica em média 36,55 minutos no sistema até que saia permanentemente. O tempo mínimo que um eixo permanece no sistema são 35,33 minutos, e máximo que este fica no sistema são 37,59 minutos. O desvio padrão obtido foi de 0,46 minutos. Case notar que o tempo médio obtido foi considerado satisfatório, considerando as características do sistema CONWIP.

Tabela 16.2 Tempo médio do eixo no sistema no cenário CONWIP

\begin{tabular}{|c|c|c|c|c|c|}
\hline Variável & Média & Mínimo & Máximo & $\begin{array}{c}\text { Desvio } \\
\text { Padrão }\end{array}$ & $\begin{array}{c}\text { Half- } \\
\text { width }\end{array}$ \\
\hline $\begin{array}{l}\text { Tempo médio no sistema } \\
\text { (minutos) }\end{array}$ & 36,55 & 35,33 & 37,59 & 0,46 & 0,10 \\
\hline
\end{tabular}

Fonte: Dados da pesquisa.

Como pode ser observado na Tabela 16.3 , o recurso mais utilizado no cenário elaborado foi o operador 5, sendo utilizado em média, 75,03\% do tempo útil de operação. Já os operadores com as menores utilizações foram o 
operador 1 e o operador 6 . Dentre os fatores que contribuem para essa menor utilização, estão: o uso da talha elétrica, pois as movimentações de eixos nas respectivas estações são realizadas por este equipamento; o tempo de processamento na estação de trabalho 6 é constante, independentemente do tipo de eixo produzido.

Tabela 16.3 Utilização dos recursos no cenário CONWIP

\begin{tabular}{|l|c|c|c|c|c|}
\hline Recurso & $\begin{array}{c}\text { Média } \\
(\%)\end{array}$ & $\begin{array}{c}\text { Mínimo } \\
(\%)\end{array}$ & $\begin{array}{c}\text { Máximo } \\
(\%)\end{array}$ & $\begin{array}{c}\text { Desvio } \\
\text { Padrão }\end{array}$ & $\begin{array}{c}\text { Half- } \\
\text { width }\end{array}$ \\
\hline Operador 1 & 49,46 & 47,76 & 51,37 & 0,75 & 0,17 \\
\hline Operador 2 & 64,73 & 62,58 & 67,49 & 1,03 & 0,24 \\
\hline Operador 3 & 64,30 & 62,05 & 66,88 & 1,04 & 0,24 \\
\hline Operador 4 & 65,59 & 64,02 & 67,71 & 0,88 & 0,20 \\
\hline Operador 5 & 75,03 & 72,64 & 78,01 & 0,99 & 0,23 \\
\hline Operador 6 & 56,56 & 55,06 & 58,56 & 0,72 & 0,17 \\
\hline
\end{tabular}

Fonte: Dados da pesquisa.

Cabe ressaltar que a medida de desempenho 'utilização dos recursos' é de importância para a análise do modelo, uma vez que a carga operacional média não deve ultrapassar $85 \%$ do tempo da jornada de trabalho. Portanto, verificouse que as porcentagens de utilização foram diferentes, mas não ultrapassaram o limite estipulado pela empresa.

A Tabela 16.4 aborda os percentuais de utilização das estações de trabalho. Foi constatado que a estação 1 possui maior utilização média, equivalente a $97,18 \%$ do tempo útil, ou seja, essa estação é utilizada praticamente durante todo o tempo da simulação. Enquanto que a estação com utilização mais baixa é a estação de trabalho 5. Em média, esta é utilizada em 57,15\% do tempo útil. Assim, esta tem uma taxa de ociosidade maior que as demais estações de trabalho. Para todas as estações de trabalho, houve variação entre os valores médios e seus mínimos e máximos, que é representado pelo desvio padrão. Cabe ressaltar que as estações de trabalho possuem capacidade unitária e que quanto maior a sua utilização, melhor para a empresa (menor ociosidade). 
Tabela 16.4 Utilização das estações de trabalho no cenário CONWIP

\begin{tabular}{|c|c|c|c|c|c|}
\hline Local & $\begin{array}{c}\text { Média } \\
(\%)\end{array}$ & $\begin{array}{l}\text { Mínimo } \\
(\%)\end{array}$ & $\begin{array}{c}\text { Máximo } \\
(\%)\end{array}$ & $\begin{array}{l}\text { Desvio } \\
\text { Padrão }\end{array}$ & $\begin{array}{l}\text { Half- } \\
\text { width }\end{array}$ \\
\hline Estação de trabalho 1 & 97,18 & 97,03 & 97,29 & 0,05 & 0,01 \\
\hline Estação de trabalho 2 & 67,28 & 65,04 & 69,90 & 1,05 & 0,24 \\
\hline Estação de trabalho 3 & 65,44 & 63,86 & 67,54 & 0,87 & 0,20 \\
\hline Estação de trabalho 4 & 76,18 & 73,77 & 79,17 & 1,00 & 0,23 \\
\hline Estação de trabalho 5 & 57,15 & 55,62 & 59,16 & 0,73 & 0,17 \\
\hline
\end{tabular}

Fonte: Dados da pesquisa.

Os valores apresentados na Tabela 16.5 mostram que, no geral, o percentual de utilização dos estoques intermediários foi baixo. No entanto, o estoque intermediário 3 foi utilizado, em média, 31,55\% do tempo útil. Mesmo assim, esta utilização foi considerada baixa. A baixa utilização dos estoques intermediários pode ser interpretada da seguinte forma: os eixos praticamente não esperam nos estoques 1, 2 e 4 para continuar o processamento na próxima estação de trabalho devido a estas estarem ocupadas.

Tabela 16.5 Utilização dos estoques intermediários no cenário CONWIP

\begin{tabular}{|l|c|c|c|c|c|}
\hline Local & $\begin{array}{c}\text { Média } \\
(\%)\end{array}$ & $\begin{array}{c}\text { Mínimo } \\
(\%)\end{array}$ & $\begin{array}{c}\text { Máximo } \\
(\%)\end{array}$ & $\begin{array}{c}\text { Desvio } \\
\text { Padrão }\end{array}$ & $\begin{array}{c}\text { Half- } \\
\text { widrh }\end{array}$ \\
\hline Estoque 1 & 0,76 & 0,53 & 0,98 & 0,10 & 0,02 \\
\hline Estoque 2 & 2,47 & 0,71 & 5,51 & 1,05 & 0,24 \\
\hline Estoque 3 & 31,55 & 27,89 & 34,12 & 1,33 & 0,31 \\
\hline Estoque 4 & 0,25 & 0,16 & 0,36 & 0,04 & 0,01 \\
\hline
\end{tabular}

Fonte: Dados da pesquisa.

Com a baixa utilização dos estoques intermediários, foi possível constatar a vantagem do sistema CONWIP exposta por Ovalle e Marquez (2003) na seção um deste artigo. Salienta-se que, se o objetivo da empresa for a redução de estoques intermediários, o CONWIP se mostrou um forte candidato para implantação na linha de produção. 


\section{Considerações finais}

Neste artigo foi simulado e analisado o desempenho do sistema de emissão de ordens CONWIP, considerando sua aplicação em uma empresa automobilística. Por meio do uso da simulação computacional, foi possível analisar o comportamento do sistema CONWIP na empresa considerada para a elaboração do cenário. Portanto, o objetivo proposto foi atingido.

Dentre os resultados alcançados na simulação, observou-se que o sistema CONWIP obteve melhor desempenho no que se refere a baixa porcentagem média de utilização de estoques intermediários. Ainda, observou-se que foram satisfatórias as porcentagens médias de utilização dos operadores e das estações de trabalho, e também quanto ao tempo médio do eixo no sistema.

Ressalta-se que foi observada a presença do seguinte trade-off: se a empresa objetiva possuir menor quantidade média de estoques intermediários e menor tempo médio no sistema, o indicado para o ambiente produtivo provavelmente será o CONWIP. No entanto, se a empresa deseja maior total médio de saídas do sistema, cabe também a análise de outras opções de sistemas de emissão de ordens, por exemplo, o kanban.

As contribuições desta pesquisa são para as áreas acadêmica e empresarial. O presente artigo divulga o tema: análise de sistemas de emissão de ordens em ambientes de manufatura. Por ser uma fonte de aplicação prática, os resultados aqui apresentados e discutidos podem auxiliar os gestores no controle da produção e, ainda, promover o interesse em se realizar um processo decisório com uso de simulação. Esta técnica foi considerada como uma ferramenta de auxílio a gestão da produção, pois foi possível analisar se o sistema é adequado à empresa antes da sua efetiva implementação.

Sugere-se, para pesquisas futuras, simular, analisar e comparar o desempenho do CONWIP com outros sistemas de emissão de ordens. Ainda, é possível realizar um estudo de escopo (scoping study) para verificar quais sistemas ainda não foram comparados na literatura.

\section{Agradecimentos}

Os autores do artigo gostariam de agradecer ao CNPq pela bolsa concedida pelo Programa Institucional de Bolsa de Iniciação Científica (PIBIC/UFG/CNPq).

\section{Referências}

BRYMAN, A. Research methods and organization studies. London: Uniwin Hyman, 1989. 224 p. 
BURBIDGE, J. L. Production Control: a universal conceptual framework. Production Planning and Control, v. 1, n.1, p. 3-16, 1990.

CHWIF, L.; MEDINA, A. C. Modelagem e Simulação de Eventos Discretos: Teoria e Aplicações. $4^{a}$ ed. Rio de Janeiro: Elsevier, 2015.

FREITAS FILHO, P. J. Introdução à modelagem e simulação de sistemas. $2^{\text {a }}$ ed. Florianópolis: Visual Books, 2008.

GONG, Q.; YANG, Y.; WANG, S. Information and decision-making delays in MRP, KANBAN and CONWIP. Int. J. Production Economics, v.156, p. 208-213, 2014.

HAN, X.; WANG, D. Optimization on RFID-enabled CONWIP control strategy for multi-echelon inventory of supply chain. In: World Congress on Intelligent Control and Automation, 12, 2016, Guilin. Proceedings... Guilin: WCICA 2016, 2016, p. 246-250.

HARRELL, C.; GHOSH, B.K.; BOWDEN, R. O. Simulation using ProModel. $3^{\text {a }}$ ed. New York: McGraw Hill, 2010.

HERER, Y. T.; MASIN, M. Mathematical programming formulation of CONWIP based production lines and relationships to MPR. Int. J. Prod. Res. v. 35, n. 4, p. 1067-1076, 1997.

HOPP, W. J.; SPEARMAN, M. L.; Factory Physics: foundations of manufacturing management. 3 ed. Chicago: Irwin/McGraw Hill, 2008.

HUANG, G.; CHEN, J.; WANG, X.; SHI, Y.; TIAN, H. From loop structure to policy-making: a CONWIP design framework for hybrid flow shop control in one-of-a-kind production environment. International Journal of Production Research. p. 1-18, September 25, 2016 a.

HUANG, G.; CHEN, J.; WANG, X.; SHI, Y. An approach of designing CONWIP loop for assembly system in one-of-a-kind production environment. International Journal of Computer Integrated Manufacturing. v. 29, n. 7, p. 805-820, July 2, 2016b.

HUANG, M.; WANG, D.; IP, W. H. Simulation study of CONWIP for a cold rolling plant. International Journal of Production Economics, v. 54, p. 257-266, 1998a. 
JAEGLER, Y.; BURLAT, P.; LAMOURI, S. The ConWip production control system: a literature review. In: International Conference on Information Systems, Logistics and Supply Chain, 6, 2016, Bordeaux. Proceedings... Bordeaux: ILS 2016, 2016.

KELTON, W. D.; SADOWSKI, R. P.; SADOWSKI, D. A. Simulation with Arena. $2^{\text {nd }}$ ed., New York: McGraw-Hill, 2002.

LAW, A. M.; KELTON, W. D. Simulation Modeling \& Analysis. 3rd edition, New York: McGraw-Hill, 2000.

LEE, H.; SEO, D. Performance evaluation of WIP-controlled line production systems with constant processing times. Computers and Industrial Engineering, v. 94, p. 138-146, 2016.

OVALLE, O. R.; MARQUEZ, A. C. Exploring the utilization of a CONWIP system for supply chain management. A comparison with fully integrated supply chains. Int. J. Production Economics, v. 83, p. 195-215, 2003.

SIPPER, D.; BULFIN JR., R. L. Production: planning, control, and integration. New York: McGraw-Hill, 1997. 630 p.

SPEARMAN, M. L.; WOODRUFF, D.; HOPP, W. J. C.: a pull alternative to kanban. International Journal of Production Research, v. 28, n.5, p. 879-894, 1990.

SPEARMAN, M. L.; ZAZANIS, M.A. Push and pull production systems: issue and comparison. Operations Research, v.40, n.3, p.521-532, 1992. 